\title{
Newly Developed Friction Tester for in situ Soft X-Ray Absorption Measurements of Frictional Engine-Oil/Metals Interfaces
}

\author{
Yasuji Muramatsu, ${ }^{* \dagger}$ Masaru OKuyama, ${ }^{* *}$ Naoko TAKahashi, ${ }^{* *}$ Toshihide OhMORI, ${ }^{* *}$ and \\ Eric M. GULLIKSON*** \\ *Graduate School of Engineering, University of Hyogo, 2167 Shosha, Himeji, Hyogo 671-2201, Japan \\ **Toyota Central R\&D Labs., Inc., 41-1 Yokomichi, Nagakute, Aichi 480-1192, Japan \\ ***Center for X-Ray Optics, Lawrence Berkeley National Laboratory, 1 Cyclotron Road, Berkeley, CA 94720, USA
}

\begin{abstract}
A novel friction tester has been developed to clarify the friction mechanism between engine oil and metals by in situloperando measurements of the X-ray absorption near edge structure (XANES) using the total electron yield (TEY) method. The tester can perform frictional motion with engine oil and metals in a vacuum chamber under a vacuum of $10^{-7}$ Torr during TEY-XANES measurements in BL-6.3.2 at the Advanced Light Source (ALS). From in situ XANES measurements in the $\mathrm{C} K$ and $\mathrm{Fe} L$ regions, the organic molecule layers at the oil/metals interfaces have been successfully observed.
\end{abstract}

Keywords Synchrotron radiation, soft X-ray absorption spectroscopy, friction, tribology

(Received June 26, 2017; Accepted August 23, 2017; Published December 10, 2017)

\section{Introduction}

Decreasing friction in automobile engines is a global issue from the viewpoints of energy conservation and $\mathrm{CO}_{2}$ exhaust reduction. Tribology research on engine systems to develop lower frictional surfaces has attracted much attention. In general, engine oil is composed of a base oil (organic polymers) and various additive compounds. Additive molecules adsorb on friction-metal surfaces. The interface structure should chemically and/or physically reduce friction. ${ }^{1}$ The friction mechanism has previously been researched by observing metal surfaces rubbed with engine oils using conventional analytical methods, such as IR (infrared spectroscopy), ${ }^{2-4}$ Raman spectroscopy, ${ }^{4-6}$ EPMA (electron probe micro analyzer), ${ }^{7,8}$ XPS (X-ray photoelectron spectroscopy), ${ }^{5-10}$ XRD (X-ray diffraction) $)^{11-13}$ and SIMS (secondary ion mass spectroscopy). ${ }^{14-16}$ However, the friction mechanism, especially the chemical states of additives/metal interfaces, has yet to be fully elucidated because engine oil components are complicated, and direct observations at the friction interface between liquid-engine-oil/ solid-metal-surfaces are experimentally difficult.

Recently, advanced analytical methods using synchrotron radiation (SR) have been applied to observe liquid-engine-oil/ solid-metal interfaces. SR-based XPS and XAS (X-ray absorption spectroscopy) have recorded the engine-oil/metals interfaces with an emphasis on the chemical analysis of metal elements, such as S, P, or Mo, ${ }^{17-26}$ because these elements can be identified in the matrices of engine-oil organic molecules. These studies are ex situ analyses in which metal surfaces rubbed with engine-oil are analyzed. However, ex situ

$\dagger$ To whom correspondence should be addressed.

E-mail: murama@eng.u-hyogo.ac.jp measurements make it difficult to directly clarify the dynamics of the frictional mechanism.

SR-based XRD has recently been applied to in situ observations of metal surfaces in frictional motions. ${ }^{27}$ In situ XRD measurements were performed using a hard X-ray SR beam, which transmitted the frictional window material of a sapphire plate, allowing phase transformations on frictional metal surfaces to be successfully observed. On the other hand, SR-based in situ observations focusing on the organic molecules at engine-oil/metals interfaces are rare due to experimental difficulties. Although soft X-ray spectroscopy is a powerful tool for the chemical analysis of organic molecules, measurements should be achieved under a high vacuum.

For in situ soft X-ray spectroscopy, the frictional motions of metals with liquid engine oil should be performed. The signals from frictional surfaces should be sufficiently detected during the frictional motions in a high-vacuum chamber. To overcome experimental difficulties, we developed a compact friction tester for in situ and/or operando soft X-ray absorption measurements of the engine-oil/metals interfaces. This paper describes the design concept of the friction tester and the first operation of in situ soft X-ray absorption measurements of engine-oil/metals interfaces.

\section{Design of the Friction Tester}

The friction tester was designed based on the following concepts:

(1) The total-electron-yield (TEY) signal from the rotating metal ring in contact with a counter metal block with engine oil under 203-MPa loading can be measured for in situloperando observations during the frictional motion of metals with engine-oil.

(2) The friction tester will be installed and operated in the 

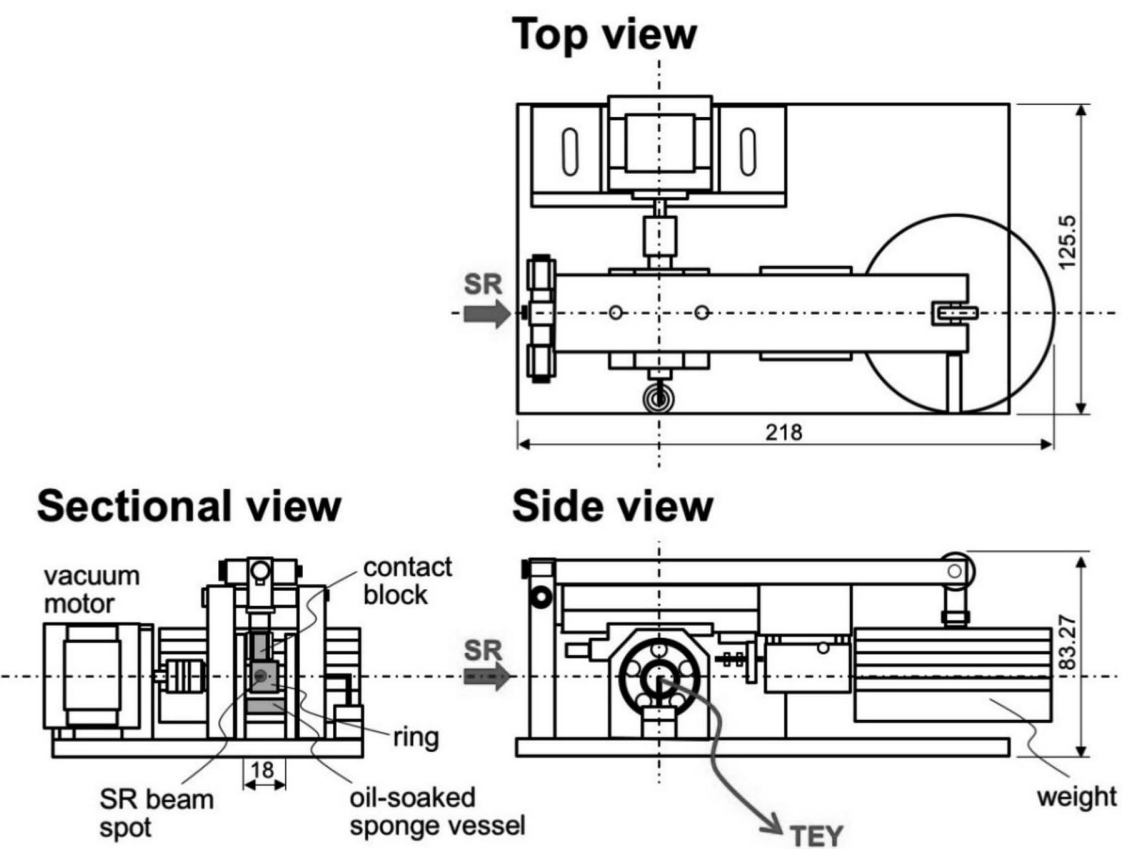

Fig. 1 Schematics of the friction tester.

vacuum measurement chamber of BL-6.3.228 at the Advanced Light Source (ALS) under a vacuum of $10^{-7}$ Torr.

(3) The rotation speed, loading pressure, and temperature of the rotating ring can be well controlled in the vacuum chamber.

Figure 1 shows a schematic of the tester. The tester unit was composed of aluminum. The tester unit was compact $(218 \times$ $125.5 \times 83.27 \mathrm{~mm}^{3}$ ), allowing it to be placed in the measurement chamber in BL-6.3.2. The rotating ring and counter block were made from SUS440C steel, which exhibits the highest hardness in stainless steels. It is well known that SUS440C steel contains $0.95-1.2 \mathrm{wt} \%$ carbon. The diameter and length of the ring were 14 and $11-12 \mathrm{~mm}$, respectively. The size of the counter block was $15.7^{\mathrm{T}} \times 10^{\mathrm{H}} \times 6.5^{\mathrm{W}} \mathrm{mm}^{3}$, where $6.5^{\mathrm{W}} \mathrm{mm}$ was the rubbing width to the ring. The ring touched an oil-soaked sponge, which contained $100-\mu \mathrm{L}$ liquid oil. Consequently, the ring and the counter block were always wet with the oil in the vacuum chamber.

The load on the ring was varied, depending on the weight of SUS304 steel. At a $1.5-\mathrm{kg}$ weight, the maximum loading to the rotating ring by the counter block was $203 \mathrm{MPa}$. The ring was rotated by a commercially available vacuum stepping motor (Kohzu Precision, 103H5508-83V2). The typical rotating speed of the ring was $300 \mathrm{~mm} / \mathrm{s}$. The TEY signal from the rotating ring was monitored from the rotating axis, which was electrically isolated from the other metal parts. The rotating speed of the ring, the temperature and the friction of the counter block were electrically controlled and monitored outside of the chamber.

Figure 2 shows photos of the friction tester installed in the vacuum chamber. A side view (a) shows the compact setting in the chamber. The rotation axis was connected to a current meter for the TEY measurements through a conducting wire. The SR beam spot (visible light) irradiated on the ring is clearly shown in the cross-sectional view (b). The beam spot size is approximately $40 \mu \mathrm{m}^{\mathrm{V}} \times 300 \mu \mathrm{m}^{\mathrm{H}}$ in the full width at half maximum (FWHM). The Au plates, which monitored beam intensity, and the highly oriented pyrolytic graphite (HOPG),
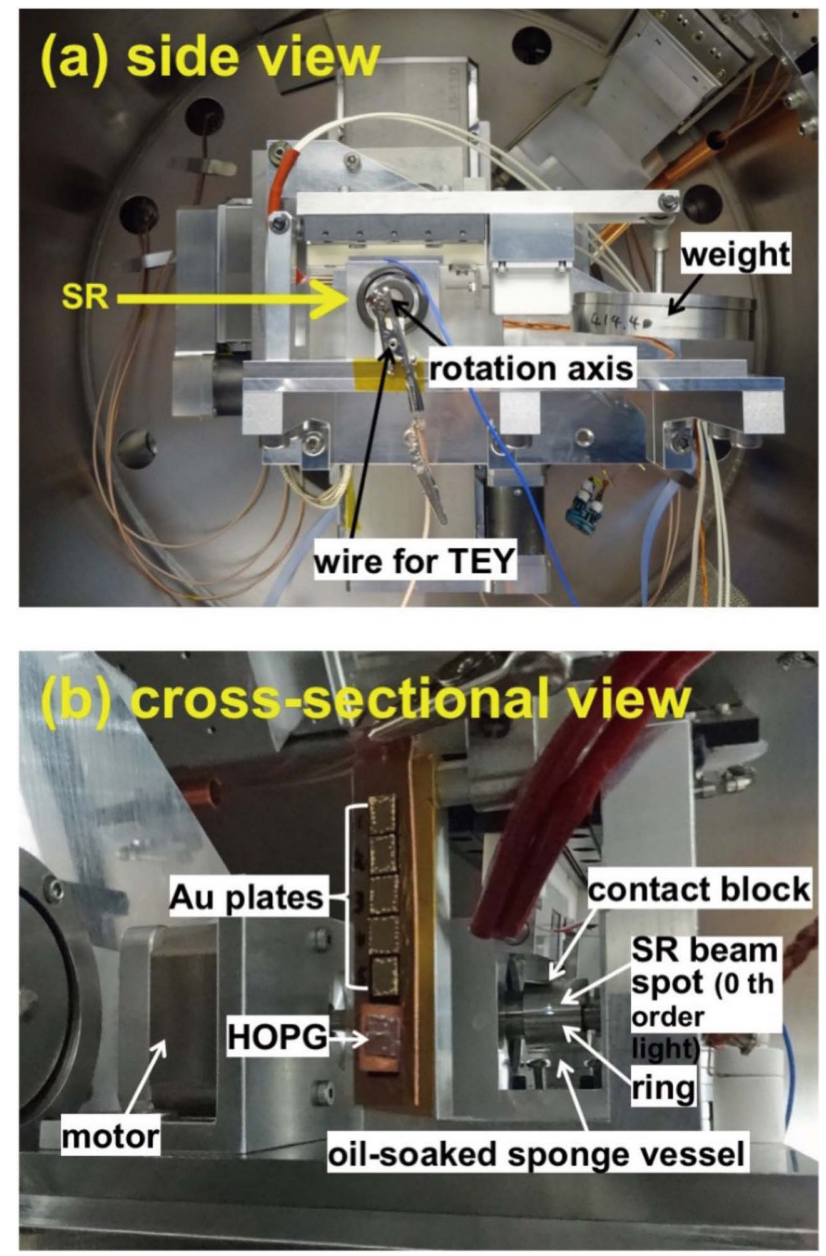

Fig. 2 Photos of the friction tester installed in the vacuum measurement chamber of BL-6.3.2. 


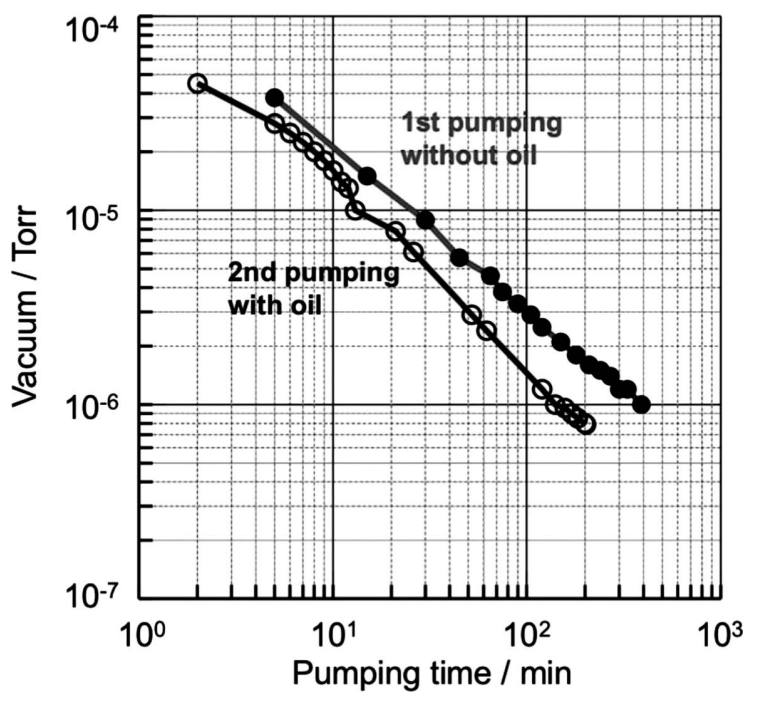

Fig. 3 Vacuum evolution while vacuum pumping the measurement chamber in which the friction tester is installed with and without oil.

which is a reference compound, were placed in front of the tester. The TEY for each sample was also measured by another conducting wire and a current meter.

\section{Vacuum Test}

The most important experiment in this study is vacuum pumping of the chamber containing the friction tester with/without liquid engine oil. Soft X-ray spectroscopic measurements should be performed under a high vacuum. Additionally, soft X-ray spectroscopy cannot be used to measure voluminous liquid oil samples because liquid oil samples may impact carbon contamination on the beamline optics. Figure 3 shows the vacuum evolution of the measurement chamber with the friction tester as a function of the pumping time. The chamber was pumped by a cryopump (Cryotorr 8 ) with a pumping speed of $4000 \mathrm{~L} / \mathrm{s}$. The first pumping without oil took approximately $400 \mathrm{~min}$ to reach a pressure on the $10^{-7}$ Torr order. After the first pumping, $100-\mu \mathrm{L}$ oil was soaked in the sponge and the chamber was pumped. Although the liquid oil was in vacuum chamber, the second pumping reached the $10^{-7}$ Torr order at around $120 \mathrm{~min}$. These vacuum pumping experiments show that the first pumping of the friction tester is critical to degas the tester components. Subsequent vacuum pumping with oil can be fairly fast because the engine-oil is essentially composed of non-volatile organic compounds. After the second pumping, the oil-coated ring was rotated for 30 min under a vacuum of $10^{-7}$ Torr. These results confirm that the friction tester with oil can be successfully operated under a pressure on the order of $10^{-7}$ Torr in the measurement chamber of BL-6.3.2.

\section{In situ XANES Measurements}

In situ XANES measurements of the rubbed ring with a counter block and engine oil were performed using the TEY method under a vacuum of $10^{-7}$ Torr. The ring was rotated at a speed of $300 \mathrm{~mm} / \mathrm{s}$, a load of $151 \mathrm{MPa}$, and at a temperature of $80^{\circ} \mathrm{C}$, which are usual conditions in such a bench test for practical automobile engines. Figure 4 shows the XANES in the Fe $L$
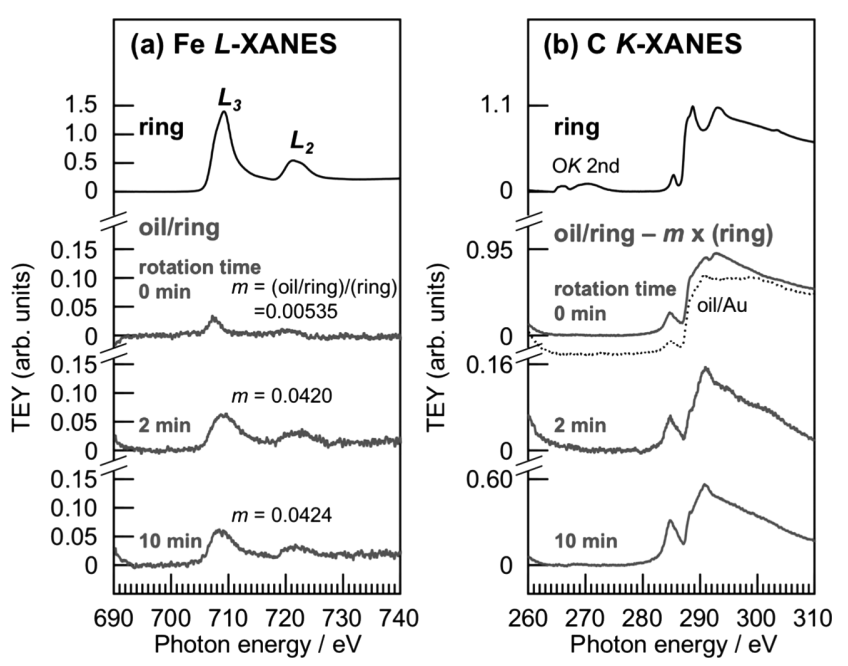

Fig. 4 In situ measured XANES in the Fe $L$ (a) and $\mathrm{C} K(\mathrm{~b})$ regions of the original ring and the oil/ring sample rotated for 0,2 , and $10 \mathrm{~min}$. Dotted line is the C $K$-XANES of oil/Au, compared to the 0 -min rotated oil/ring.

and $\mathrm{C} K$ regions of the original ring and the ring rotated for 0 , 2, and $10 \mathrm{~min}$ with oil (denoted by oil/ring in the figure). Each spectrum was acquired just after rotation in vacuum. Thus, they are in situ XANES.

In the Fe $L$-XANES of the oil/ring samples, $\mathrm{Fe} L_{2,3}$ peaks were small in 0 -min rotation. This means that the oil layer initially coated on the ring was sufficiently thick to observe the ring surface by the TEY method. However, Fe $L_{2,3}$ peaks were clearly observed in the 2- and 10-min rotated samples, indicating that the oil layer became thinner by rotation, and the interface between the oil and ring was observed by TEY. The contribution factor $m$ can be defined from the Fe $L_{3}$ peak height, $I_{\mathrm{FeL}}$, between the oil/ring and the original ring as $m=I_{\mathrm{FeL}}(\mathrm{oil} / \mathrm{ring}) / I_{\mathrm{FeL}}($ ring $) .{ }^{29}$ Therefore, $m$ means the contribution ratio of the ring to the oil/ ring in XANES.

The measured $m$ factors of the oil/ring sample were described in the Fe L-XANES. The initial oil/ring sample $(0 \mathrm{~min})$ exhibited $m=0.00535$, implying that the contribution from the ring was approximately $0.5 \%$. On the other hand, the oil/ring samples rotated for 2 and $10 \mathrm{~min}$ exhibited $m$ values as large as $\sim 0.04$. This means that the contribution from the ring increased to $4 \%$ due to the thinner oil layers due to the rotation. Additionally, the oil layer rapidly became thinner due to the initial rotation within 2 min. In C $K$-XANES, carbon was clearly observed in the original ring sample because the SUS440C steel contained carbon. The C $K$-XANES of the oil/ ring sample was described by eliminating the contribution from the ring, such as (oil/ring) $-m \times($ ring), where $m$ was obtained from the Fe $L$-XANES. The C $K$-XANES profile of the oil/ring sample in the 0 -min rotation differed from that of the original ring, implying that a thick oil layer could be initially coated on the ring. The 0 -min rotation profile is also in agreement with the $\mathrm{C} K$-XANES of oil coated on a clean $\mathrm{Au}$ substrate. Therefore, the 0 -min rotation profile is regarded as being the typical XANES of the thick oil layer. However, the profile changed in the 2- and 10-min rotated oil/ring sample. It exhibited a sharp peak at $285 \mathrm{eV}$, a shoulder at $288 \mathrm{eV}$, and a peak at $291 \mathrm{eV}$. As mentioned above in the Fe $L$-XANES, the spectra should also show the thinner oil layers that include the oil/ring interface. Hence, the C $K$-XANES of the oil/ring in the 
2- and 10-min rotations should reflect the chemical states of oil at the interface. The chemical states differed from that of the original oil molecules. The in situ observed chemical states at the oil/ring interfaces should be formed by friction.

However, the $m$ values in the present in situ measurements $(\sim 0.04)$ are smaller than the previous values $(\sim 0.7)$ obtained by ex situ measurements. ${ }^{29}$ In ex situ measurements, excess oil layers were removed by washing with organic solvent after frictions. Thus, the ex situ measured oil layers were sufficiently thin to observe the oil/metal interfaces. Although it is hard to estimate the oil layer thickness, the smaller $m$ values in the present in situ measurements mean that the oil layers are approximately 10-times thicker than those in the ex situ measurements. Therefore, C $K$-XANES of the present in situ measurements may fairly include information concerning the upper oil layers on the interfaces. Additionally, TEY intensity of $\mathrm{C} K$-XANES was unstable during ring rotations, as shown in Fig. 4(b); the TEY intensity of 10-min rotation is 4-times higher than that of 2-min rotation. Spatial nonuniformity of the frictional motions may take place at the oil/ring interfaces. Hence, improvements to form thinner oil layers and to perform stable frictional motions should be necessary to clearly observe the oil/ring interfaces.

\section{Conclusions}

In summary, we developed a friction tester for in situ and/or operando TEY-XANES measurements at engine-oil/metals interfaces during frictional motion in a vacuum measurement chamber. The first operations of the tester were demonstrated at BL-6.3.2/ALS. Prior to frictional operations, vacuum pumping of the tester with liquid oil was performed for $10 \mathrm{~h}$ in the measurement chamber. A vacuum of $10^{-7}$ Torr was achieved. In situ TEY-XANES measurements in the $\mathrm{Fe} L$ and $\mathrm{C} K$ regions at the frictional oil/ring interface were successfully performed. By monitoring the $\mathrm{Fe} L_{3}$ peak intensity, the contribution ratio in XANES of the ring onto the oil/ring sample was estimated as the contribution factor, $m$. Considering the measured $m, \mathrm{C}$ $K$-XANES of thin oil layers including the oil/metals interfaces was obtained in the 2- and 10-min rotations.

Such measurements using the friction tester are the first in situ experiments in tribology research and in soft X-ray spectroscopy. To perform operando measurements using the frictional tester in the near future, we are now improving the measurement conditions to control oil layer thickness and the stable ring rotation system.

\section{Acknowledgements}

The authors express their gratitude to Dr. Mamoru Tohyama of Toyota Central R\&D Laboratories for his helpful discussion on the frictional oil/metals interfaces.

\section{References}

1. R. M. Mortier, M. F. Fox, and S. T. Orszulik, "Chemistry and Technology of Lubricants", 3rd ed., 2010, Springer, Dordrecht, Heidelberg, London, New York.
2. R. Lu, S. Mori, H. Tani, N. Tagawa, and S. Koganezawa, Tribology Intl., 2017, 113, 36.

3. M. J. Adams, M. J. Romeo, and P. Rawson, Talanta, 2007, 73, 629 .

4. F. M. Salehi, A. Morina, and A. Neville, Tribology Int., 2017, 115, 285.

5. A. Datta, A. Sadhu, B. Sen, M. Kaur, R. Sharma, S. C. Das, and S. Bhattacharyya, Corrosion Science, 2013, 73, 356.

6. L. Dobrenizki, S. Tremmel, S. Wartzack, D. C. Hoffmann, T. Brögelmann, K. Bobzin, N. Bagcivan, Y. Musayev, and T. Hosenfeldt, Surf. Coatings Technol., 2016, 308, 360.

7. J. Zhang, W. Liu, Q. Xue, and Q. Wang, Wear, 1998, 216, 35.

8. M. Gautam, K. Chitoor, M. Durbha, and J. C. Summers, Tribology Intl., 1999, 32, 687.

9. L. Zhu, X. Wu, G. Zhao, and X. Wang, Tribology Intl., 2016, 104, 383.

10. P. Forsberg, F. Gustavsson, V. Renman, A. Hieke, and S. Jacobson, Wear, 2013, 304, 211.

11. Z. Meng, Y. Li, Y. Yang, Z.-Q. Xu, B. Shi, and S.-L. Zhao, J. Petroleum Sci. Eng., 2015, 128, 140.

12. B. Karpuschewski, F. Welzel, K. Risse, and M. Schorgel, Procedia CIRP, 2016, 45, 87.

13. A. L. Bandeira, R. Trentin, C. Aguzzoli, M. E. H. Maia da Costa, A. F. Michels, I. J. R. Baumvol, M. C. M. Farias, and C. A. Figueroa, Wear, 2013, 301, 786.

14. A. Murase and T. Ohmori, Appl. Surf. Sci., 2003, 203-204, 586.

15. A. Murase, H. Mori, and T. Ohmori, Appl. Surf. Sci., 2008, 255, 1494.

16. T. Kubo, H. Nanao, S. Mori, Y. Enomoto, H. Nie, and H. Nomura, Wear, 2010, 268, 1225.

17. Z. Yin, M. Kasrai, G. M. Bancroft, K. F. Laycock, and K. H. Tan, Tribology Intl., 1993, 26, 383.

18. M. L. S. Fuller, M. Kasrai, G. M. Bancroft, K. Fyfe, and K. H. Tan, Tribology Intl., 1998, 31, 627.

19. J. N. Cutler, J. H. Sanders, P. J. John, G. DeStasio, B. Gilbert, and K. Tan, Wear, 1999, 236, 165.

20. M. Najman, M. Kasrai, G. M. Bancroft, and R. Davidson, Tribology Intl., 2006, 39, 342.

21. K. Fan, J. Li, H. Ma, H. Wu, T. Ren, M. Kasrai, and G. M. Bancroft, Tribology Intl., 2008, 41, 1226.

22. G. Nehme, R. Mourhatch, and P. B. Aswath, Wear, 2010, $268,1129$.

23. B. Kim, R. Mourhatch, and P. B. Aswath, Wear, 2010, 268 , 579.

24. L. G. Yu, E. S. Yamaguchi, M. Kasrai, and G. M. Bancroft, Tribology Intl., 2011, 44, 692.

25. H. Wu, Y. G. Wang, and T. H. Ren, Tribology Intl., 2012, 49, 90.

26. Z. Li, X. Li, Y. Zhang, T. Ren, Y. Zhao, X. Zeng, and E. Heide, Appl. Surf. Sci., 2014, 308, 91.

27. S. Kajita, K. Yagi, T. Izumi, J. Koyamachi, M. Tohyama, K. Saito, and J. Sugimura, Tribol. Lett., 2015, 57, 361.

28. J. H. Underwood, E. M. Gullikson, M. Koike, P. J. Batson, P. E. Denham, K. D. Franck, R. E. Tackaberry, and W. F. Steele, Rev. Sci. Instrum., 1996, 67, 3372.

29. Y. Muramatsu, K. Nambu, N. Takahashi, M. Okuyama, N. Isomura, M. Toyama, Y. Kimoto, H. Omori, and E. M. Gullikson, Adv. X-Ray Chem. Anal., Japan, 2017, 48, 327. 